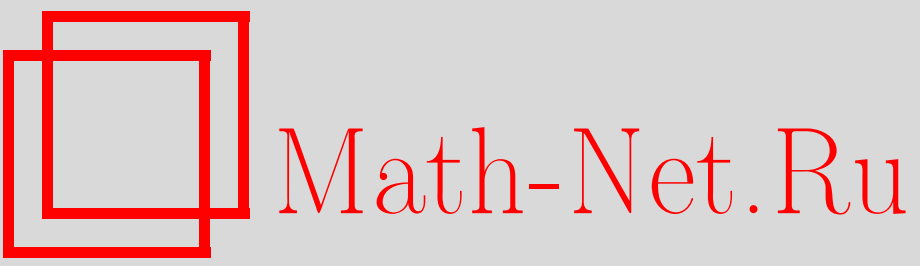

М. В. Половинкина, И. П. Половинкин, О свойствах римановых метрик, связанных с В-эллиптическими операторами, Итоги науки и техн. Сер. Соврем. мат. и ее прил. Темат. обз., 2019, том 170, 31-37

DOI: https://doi.org/10.36535/0233-6723-2019-170-31-37

Использование Общероссийского математического портала Math-Net.Ru подразумевает, что вы прочитали и согласны с пользовательским соглашением

http: //www.mathnet.ru/rus/agreement

Параметры загрузки:

IP : 54.205 .225 .156

26 апреля 2023 г., 03:10:04 


\title{
О СВОЙСТВАХ РИМАНОВЫХ МЕТРИК, СВЯЗАННЫХ С В-ЭЛЛИПТИЧЕСКИМИ ОПЕРАТОРАМИ
}

\author{
(c) 2019 г. М. В. ПОЛОВИНКИНА, И. П. ПОЛОВИНКИН
}

\begin{abstract}
АннотАция. Рассмотрена риманова метрика, в которой оператор Лапласа-Бельтрами с точностью до множителя совпадает с $B$-эллиптическим оператором.

Ключевые слова: $B$-эллиптический оператор, риманова метрика, оператор Лапласа-Бельтрами, группа изометрий, условия Киллинга, геометрия Лобачевского.
\end{abstract}

\section{ON PROPERTIES OF RIEMANNIAN METRICS ASSOCIATED WITH $B$-ELLIPTIC OPERATORS}

\section{(c) 2019 M. V. POLOVINKINA, I. P. POLOVINKIN}

\begin{abstract}
In this paper, we consider a Riemannian metric in which the Laplace-Beltrami operator coincides with a $B$-elliptic operator up to a factor.

Keywords and phrases: B-elliptic operator, Riemannian metric, Laplace-Beltrami operator, isometry group, Killing conditions, Lobachevsky geometry.
\end{abstract}

AMS Subject Classification: 35J15

1. $\quad$ K-Однородная метрика. Пусть

$$
\gamma=\left(\gamma_{1}, \ldots, \gamma_{n}\right)
$$

где $\gamma_{i}, i=1 \ldots, n,-$ фиксированные числа, одновременно не равные нулю, т.е. такие, что

$$
\sum_{i=1}^{n} \gamma_{i}^{2}>0
$$

Обозначим символом $\mathbb{R}_{+}^{n}$ множество таких $x=\left(x_{1}, \ldots, x_{n}\right) \in \mathbb{R}^{n}$, что $x_{i} \in \mathbb{R}$, если $\gamma_{i}=0$, $x_{i} \in(0,+\infty)$, если $\gamma_{i} \neq 0$. Переменную $x_{i}$ с номером $i$, для которого $\gamma_{i} \neq 0$, принято называть особой. Как обычно, будем пользоваться обозначением

$$
(x)^{\gamma}=\prod_{i=1}^{n} x_{i}^{\gamma_{i}}, \quad x=\left(x_{1}, \ldots, x_{n}\right) \in \mathbb{R}_{+}^{n} .
$$

Пусть функция $u(x)$ дважды непрерывно дифференцируема в $\mathbb{R}_{+}^{n}$.

Определим оператор $\Delta_{B_{\gamma}}$ формулой

$$
\Delta_{B_{\gamma}} u=\sum_{i=1}^{n} \frac{\partial^{2} u}{\partial x_{i}^{2}}+\sum_{i=1}^{n} \frac{\gamma_{i}}{x_{i}} \frac{\partial u}{\partial x_{i}} .
$$

Операторы вида (1) изучались И. А. Киприяновым и его учениками (см. [4-6]). 
Поставим задачу найти такую положительно определенную на $\mathbb{R}_{+}^{n}$ симметричную квадратичную форму (метрику)

$$
d s^{2}=\sum_{i=1}^{n} \sum_{j=1}^{n} g_{i j} d x_{i} d x_{j}
$$

чтобы соответствующий этой метрике оператор Лапласа-Бельтрами (см. [8])

$$
\Delta_{\omega}=\frac{1}{\sqrt{|g|}} \sum_{i=1}^{n} \frac{\partial}{\partial x_{i}} \sum_{j=1}^{n} g^{i k} \sqrt{|g|} \frac{\partial}{\partial x_{k}}
$$

с точностью до множителя совпадал с оператором $\Delta_{B_{\gamma}}$. Здесь функции $g^{i j}, i, j=1, \ldots, n,-$ элементы матрицы $\left\|g^{i j}\right\|$, обратной к матрице $\left\|g_{i j}\right\|$ (ковариантный метрический тензор),

$$
g=\operatorname{det}\left\|g_{i j}\right\| .
$$

Изучение свойств эллиптических дифференциальных операторов с помощью римановой метрики имеет давние корни (см., например, $[2,7])$.

Теорема 1. При $n \geqslant 3$ элементы искомой матрицы $\left\|g_{i j}\right\|$ задаются с помощью формул

$$
g_{i j}=\delta_{i j} \prod_{i=1}^{n} x_{i}^{K_{i}}=\delta_{i j} x^{K}, \quad i, j=1, \ldots, n, \quad K=\left(K_{1}, \ldots, K_{n}\right),
$$

где $\delta_{i j}-$ символ Кронекера,

$$
K_{i}=\frac{2}{n-2} \gamma_{i}
$$

Доказательство. Действительно, поскольку $g_{i j}=0$ при $i \neq j$, подставляя (3) в $(2)$, получим:

$$
\Delta_{\omega} u=\frac{1}{\sqrt{|g|}} \sum_{k=1}^{n} \frac{\partial}{\partial x_{k}}\left(g^{k k} \sqrt{|g|} \frac{\partial u}{\partial x_{k}}\right),
$$

где

$$
\begin{gathered}
|g|=g=x^{n K}=\prod_{i=1}^{n} \prod_{i=1}^{N} n x_{i}^{K_{i}}=\prod_{i=1}^{n} x_{i}^{2 n \gamma_{i} /(n-2)}, \\
g^{k k}=x^{-K}=\prod_{i=1}^{n} x_{i}^{-2 \gamma_{i} /(n-2)} .
\end{gathered}
$$

Учитывая (6) и (7), можно переписать (5) в следующем виде:

$$
\begin{aligned}
& \Delta_{\omega} u=\frac{1}{x^{n K / 2}} \sum_{j=1}^{n} \frac{\partial}{\partial x_{j}}\left(x^{-K} x^{K n / 2} \frac{\partial u}{\partial x_{j}}\right)= \\
&=x^{-K} \sum_{j=1}^{n} \frac{\partial^{2} u}{\partial x_{j}^{2}}+x^{-K n / 2} \sum_{j=1}^{n} \frac{\partial u}{\partial x_{j}}\left(\prod_{l=1}^{n} x_{l}^{K_{l}(n-2) / 2}\right) \frac{\partial u}{\partial x_{j}}= \\
&=x^{-K} \sum_{j=1}^{n} \frac{\partial^{2} u}{\partial x_{j}^{2}}+x^{-K n / 2} \sum_{j=1}^{n} \prod_{l=1}^{n} x_{l}^{K}(n-2) / 2 \frac{K_{j}(n-2)}{2} x_{j}^{-1} \frac{\partial u}{\partial x_{j}}= \\
&=x^{-K} \sum_{j=1}^{n} \frac{\partial^{2} u}{\partial x_{j}^{2}}+x^{-K} \sum_{j=1}^{n} \frac{K_{j}(n-2)}{2 x_{j}} \frac{\partial u}{\partial x_{j}}=x^{-K} \Delta_{B_{\gamma}} u
\end{aligned}
$$

то есть

$$
\Delta_{\omega} u=x^{-K} \Delta_{B_{\gamma}} u
$$

что и требовалось доказать. 
Множество $\mathbb{R}_{+}^{n}$, снабженное римановой метрикой

$$
d s^{2}=x^{K} \sum_{i=1}^{n} d x_{i}^{2}, \quad K \in \mathbb{R},
$$

будем рассматривать как риманово пространство; обозначим его через $K I_{n}$, а метрику (9) будем называть $K$-однородной метрикой.

Теорема 2. При $n=2$ задача о нахождении метрики, удовлетворяющей равенству (8), не имеет решения.

Доказательство. Введем обозначения $E=g_{11}, F=g_{12}=g_{21}, G=g_{22}$. Тогда

$$
g=\operatorname{det}\left\|g_{i j}\right\|=E G-F^{2}, \quad g^{i j}=(-1)^{i+j} \frac{g_{i j}}{E G-F^{2}} .
$$

Отсюда

$$
\Delta_{\omega} u=\frac{G}{|g|} \frac{\partial^{2} u}{\partial x_{1}^{2}}+\frac{E}{|g|} \frac{\partial^{2} u}{\partial x_{2}^{2}}-2 \frac{F}{|g|} \frac{\partial^{2} u}{\partial x_{1} \partial x_{2}}+\Phi\left(\frac{\partial u}{\partial x_{1}}, \frac{\partial u}{\partial x_{2}}\right),
$$

где через $\Phi$ обозначено слагаемое, зависящее только от производных первого порядка функции $u$. Для того, чтобы выражение (10) совпадало с точностью до множителя с (1), необходимо выполнение условия $F \equiv 0$, что повлечет за собой равенства

$$
g=E G, \quad g^{11}=\frac{1}{E}, \quad g^{22}=\frac{1}{G}, \quad g_{12}=g_{21}=g^{12}=g^{21}=0 .
$$

Отсюда

$$
\begin{aligned}
\Delta_{\omega} u=\frac{1}{\sqrt{|E G|}}\left(\frac{\partial}{\partial x_{1}}\left(\sqrt{\left|\frac{G}{E}\right|} \frac{\partial u}{\partial x_{1}}\right)+\frac{\partial}{\partial x_{2}}\right. & \left.\left(\sqrt{\left|\frac{E}{G}\right|} \frac{\partial u}{\partial x_{2}}\right)\right)= \\
& =\frac{1}{E} \frac{\partial^{2} u}{\partial x_{1}^{2}}+\frac{1}{G} \frac{\partial^{2} u}{\partial x_{2}^{2}}+\frac{\partial}{\partial x_{1}} \sqrt{\left|\frac{G}{E}\right|} \frac{\partial u}{\partial x_{1}}+\frac{\partial}{\partial x_{2}} \sqrt{\left|\frac{E}{G}\right|} \frac{\partial u}{\partial x_{2}} .
\end{aligned}
$$

Первые два слагаемых должны иметь одинаковые коэффициенты, откуда $E=G$. Но тогда два последних слагаемых равны нулю, что и означает невозможность подобрать искомую метрику при $n=2$.

2. Исследование изометрических преобразований для $K$-однородной метрики. Необходимым и достаточным условием того, чтобы однопараметрическая группа $G$ с инфинитезимальным оператором

$$
X=\sum_{i=1}^{n} \xi_{i}(x) \frac{\partial}{\partial x_{i}}
$$

была группой изометрий, является выполнение условий Киллинга

Очевидно, что

$$
\sum_{s=1}^{n}\left(\xi_{s} \frac{\partial g_{i j}}{\partial x_{s}}+g_{i s} \frac{\partial \xi_{s}}{\partial x_{j}}+g_{j s} \frac{\partial \xi_{s}}{\partial x_{i}}\right)=0, \quad i, j=1, \ldots, n .
$$

Поэтому уравнения Киллинга примут вид

$$
\frac{\partial g_{i j}}{\partial x_{s}}=\delta_{i j} \frac{K_{s} x^{K}}{x_{s}}
$$

$$
\sum_{s=1}^{n}\left(\delta_{i j} \xi_{s} K_{s} x^{K-1}+x^{K}\left(\frac{\partial \xi_{i}}{\partial x_{j}}+\frac{\partial \xi_{j}}{\partial x_{i}}\right)\right)=0, \quad i, j=1, \ldots, n .
$$

Произведя суммирование и сократив на $x_{K}$, мы получим

$$
\delta_{i j} \sum_{s=1}^{N} \frac{\xi_{s} K_{s}}{x_{s}}+\frac{\partial \xi_{i}}{\partial x_{j}}+\frac{\partial \xi_{j}}{\partial x_{i}}=0, \quad i, j=1, \ldots, n .
$$


При $i \neq j$ уравнение (11) можно записать в виде

$$
\frac{\partial \xi_{i}}{\partial x_{j}}+\frac{\partial \xi_{j}}{\partial x_{i}}=0, \quad i, j=1, \ldots, n, \quad i \neq j .
$$

При $i=j$ уравнение (11) можно записать в виде

$$
2 \frac{\partial \xi_{j}}{\partial x_{j}}+\sum_{s=1}^{n} \frac{K_{s} \xi_{s}}{x_{s}}=0, \quad i=1, \ldots, n .
$$

Вектор

$$
\xi=\left(\xi_{1}, \ldots, \xi_{n}\right), \quad \xi_{j}=C x^{p} x_{j}
$$

где

$$
p=\left(p_{1}, \ldots, p_{n}\right), \quad p_{1}=p_{2}=\cdots=p_{n}=\beta=-\sum_{l=1}^{n} \frac{K_{l}}{2}-1,
$$

является решением системы (13), в чем можно убедиться непосредственной проверкой. Подставив представление (14) с учетом (15) в уравнения (12), мы получим

$$
0 \equiv \frac{\partial \xi_{i}}{\partial x_{j}}+\frac{\partial \xi_{j}}{\partial x_{i}}=\beta x^{p}\left(\frac{x_{i}}{x_{j}}+\frac{x_{j}}{x_{i}}\right), \quad i, j=1, \ldots, n, \quad i \neq j .
$$

Отсюда получаем

$$
p_{1}=p_{2}=\cdots=p_{n}=\beta=-\sum_{l=1}^{n} \frac{K_{l}}{2}-1=0,
$$

или, что то же самое,

$$
\sum_{l=1}^{n} K_{l}=-2
$$

а учитывая (4),

$$
\sum_{i=1}^{n} \gamma_{i}=2-N
$$

3. Характеристики $K$-однородной метрики в случае одной особой переменной. Один из случаев выполнения условия (16), или, что то же, (18), хорошо известен. Пространство $K I_{n}$ представляет собой модель Пуанкаре $n$-мерной геометрии Лобачевского. Далее мы рассмотрим случай $\gamma_{1}=\gamma_{2}=\cdots=\gamma_{n-1}=0, \gamma_{n} \neq 0$. Метрика (3) теперь будет иметь вид

$$
g_{i j}=\delta_{i j} x_{n}^{K}, \quad i, j=1, \ldots, n,
$$

где $\delta_{i j}$ - символ Кронекера и

$$
K=\frac{2}{n-2} \gamma
$$

Непосредственным вычислением устанавливаются следующие факты.

Теорема 3. Символы Кристоффеля первого рода, соответствующие метрике (9), имеют вид

$$
\Gamma_{i j, k}=\frac{1}{2} K x_{n}^{K-1}\left(\delta_{i k} \delta_{j n}+\delta_{j k} \delta_{i n}-\delta_{i j} \delta_{k n}\right) .
$$

Доказательство. Из определения символов Кристоффеля первого рода, учитывая (19)-(20), получим:

$$
\Gamma_{i j, k}=\frac{1}{2}\left(\frac{\partial g_{i k}}{\partial x_{j}}+\frac{\partial g_{j k}}{\partial x_{i}}-\frac{\partial g_{i j}}{\partial x_{k}}\right)=\frac{1}{2}\left(\delta_{i k} \delta_{j n} K x_{n}^{K-1}+\delta_{j k} \delta_{i n} K x_{n}^{K-1}-\delta_{i j} \delta_{k n} K x_{n}^{K-1}\right) .
$$

Теорема 4. Символь Кристофбеля второго рода, соответствующие метрике (9), имеют вид

$$
\Gamma_{i j}^{k}=\frac{K}{2 x_{n}}\left(\delta_{i k} \delta_{j n}+\delta_{j k} \delta_{i n}-\delta_{i j} \delta_{k n}\right) .
$$


Доказательство. Из определения символов Кристоффеля второго рода и предыдущей теоремы получаем:

$$
\begin{aligned}
& \Gamma_{i j}^{k}=\sum_{h=1}^{n} g^{k h} \Gamma_{i j, h}=\frac{K}{2} \sum_{h=1}^{n} \delta_{k h} x_{n}^{-K} x_{n}^{K-1}\left(\delta_{i h} \delta_{j n}+\delta_{j h} \delta_{i n}-\right.\left.\delta_{i j} \delta_{h n}\right)= \\
&=\frac{K}{2 x_{n}} \sum_{h=1}^{n}\left(\delta_{k i} \delta_{j n}+\delta_{k j} \delta_{i n}-\delta_{i j} \delta_{k n}\right) .
\end{aligned}
$$

Теорема 5. Компоненты тензора Римана, соответствующего метрике (9), имеют вид

$$
R_{i j k}^{l}=\left(\frac{K^{2}}{4 x_{n}^{2}}-\frac{K}{2 x_{n}^{2}}\right)\left(\delta_{l i} \delta_{i n} \delta_{k n}+\delta_{i k} \delta_{j n} \delta_{l n}-\delta_{i j} \delta_{k n} \delta_{l n}-\delta_{l k} \delta_{i n} \delta_{j n}\right)+\frac{K^{2}}{4 x_{n}^{2}}\left(\delta_{i j} \delta_{l k}-\delta_{i k} \delta_{l j}\right) .
$$

Доказательство. По определению компоненты тензора Римана вычисляются по формулам

$$
R_{i j k}^{l}=\frac{\partial \Gamma_{i k}^{l}}{\partial x_{j}}-\frac{\partial \Gamma_{i j}^{l}}{\partial x_{k}}+\sum_{m=1}^{n}\left(\Gamma_{i k}^{m} \Gamma_{m j}^{l}-\Gamma_{i j}^{m} \Gamma_{m k}^{l}\right)
$$

Вычислим входящие в эти формулы частные производные. Имеем

откуда

$$
\frac{\partial \Gamma_{i j}^{k}}{\partial x_{s}}=-\frac{K}{2 x_{n}^{2}} \delta_{s n}\left(\delta_{k i} \delta_{j n}+\delta_{k j} \delta_{i n}-\delta_{i j} \delta_{k n}\right)
$$

$$
\frac{\partial \Gamma_{i k}^{l}}{\partial x_{j}}=-\frac{K}{2 x_{n}^{2}} \delta_{j n}\left(\delta_{l i} \delta_{k n}+\delta_{l k} \delta_{i n}-\delta_{i k} \delta_{l n}\right), \quad \frac{\partial \Gamma_{i j}^{l}}{\partial x_{k}}=-\frac{K}{2 x_{n}^{2}} \delta_{k n}\left(\delta_{l i} \delta_{j n}+\delta_{l j} \delta_{i n}-\delta_{i j} \delta_{l n}\right) .
$$

Поэтому

$$
\frac{\partial \Gamma_{i}^{l}}{\partial x_{j}}-\frac{\partial \Gamma_{i j}^{l}}{\partial x_{s}}=-\frac{K}{2 x_{n}^{2}}\left(\delta_{j n} \delta_{l k} \delta_{i n}-\delta_{j n} \delta_{i k} \delta_{l n}-\delta_{k n} \delta_{l j} \delta_{i n}+\delta_{k n} \delta_{i j} \delta_{l n}\right) .
$$

Вычислим теперь последнее слагаемое в определении. Учитывая теорему 4, находим

$$
\begin{aligned}
& \sum_{m=1}^{n}\left(\Gamma_{i k}^{m} \Gamma_{m j}^{l}-\Gamma_{i j}^{m} \Gamma_{m k}^{l}\right)= \\
& \quad=\frac{K^{2}}{4 x_{n}^{2}} \sum_{m=1}^{n}\left(\delta_{m i} \delta_{k n} \delta_{l m} \delta_{j n}+\delta_{m i} \delta_{k n} \delta_{l j} \delta_{m n}-\delta_{m i} \delta_{k n} \delta_{m j} \delta_{l n}+\delta_{m k} \delta_{i n} \delta_{l m} \delta_{j n}+\right. \\
& +\delta_{m k} \delta_{i n} \delta_{l j} \delta_{m n}-\delta_{m k} \delta_{i n} \delta_{m j} \delta_{l n}-\delta_{i k} \delta_{m n} \delta_{l m} \delta_{j n}-\delta_{i k} \delta_{m n} \delta_{l j} \delta_{m n}+\delta_{i k} \delta_{m n} \delta_{m j} \delta_{l n}- \\
& -\delta_{m i} \delta_{j n} \delta_{l m} \delta_{k n}-\delta_{m i} \delta_{j n} \delta_{l k} \delta_{m n}+\delta_{m i} \delta_{j n} \delta_{m k} \delta_{l n}-\delta_{m j} \delta_{i n} \delta_{l m} \delta_{k n}-\delta_{m j} \delta_{i n} \delta_{l k} \delta_{m n}+ \\
& \left.\quad+\delta_{m j} \delta_{i n} \delta_{m k} \delta_{l n}+\delta_{i j} \delta_{m n} \delta_{l m} \delta_{k n}+\delta_{i j} \delta_{m n} \delta_{l k} \delta_{m n}-\delta_{i j} \delta_{m n} \delta_{m k} \delta_{l n}\right) .
\end{aligned}
$$

Отсюда, учитывая свойства символа Кронекера, в частности, формулы $\delta_{i l}=\delta_{l i}, \sum_{m=1}^{n} \delta_{m i} \delta_{l m}=\delta_{i l}$, после тождественных преобразований получаем утверждение теоремы.

Теорема 6. Компоненты тензора Риччи, соответствующего метрике (9), имеют вид

$$
R_{i j}=\frac{K}{4 x_{n}^{2}}\left((K-2)(2-n) \delta_{i n} \delta_{j n}+(K(n-2)+2) \delta_{i j}\right) .
$$

Доказательство. Непосредственно из определения компонентов тензора Риччи

$$
R_{i j}=\sum_{k=1}^{n} R_{i j k}^{k}
$$

после тождественных преобразований приходим к справедливости теоремы. 
Теорема 7. Кривизна пространства $K I_{n}$ вычисляется по формуле

$$
R=\frac{K n(n-2)}{x_{n}^{K+2}}=\frac{2 \gamma n}{x_{n}^{(2 \gamma+2 n-4) /(n-2)}} .
$$

Доказательство. Из определения кривизны

$$
R=\sum_{i=1}^{n} \sum_{j=1}^{n} g^{i j} R_{i j}
$$

приходим к утверждению теоремы, производя суммирование и тождественные преобразования.

Замечание 1. Никакая дважды непрерывно дифференцируемая замена переменных не приведет уравнение вида

к уравнению вида

$$
\Delta_{B_{\gamma}} u \equiv \sum_{k=1}^{n} \frac{\partial^{2} u}{\partial x_{k}^{2}}+\frac{\gamma}{x_{n}} \frac{\partial u}{\partial x_{n}}=f, \quad \gamma \neq 0,
$$

$$
\Delta u \equiv \sum_{k=1}^{n} \frac{\partial^{2} u}{\partial x_{k}^{2}}=f .
$$

Действительно, если бы такая замена существовала, то это означало бы, что существует координатное преобразование, переводящее метрику с ненулевой кривизной в метрику с нулевой кривизной, что невозможно.

\section{4. Исследование геодезических линий для $K$-однородной метрики.}

Теорема 8. Система уравнений геодезических пространства $K I_{n}$ сводится к системе первого порядка

$$
\frac{d x_{k}}{d s}=\frac{C_{k}}{x_{n}^{K}}, \quad k=1, \ldots, n-1, \quad\left(\frac{d x_{n}}{d s}\right)^{2}=\frac{C_{n}}{x_{n}^{K}}-\frac{B^{2}}{x_{n}^{2 K}},
$$

əде $B=\sqrt{\sum_{k=1}^{n-1} C_{k}^{2}}$.

Доказательство. Система уравнений, которым удовлетворяют геодезические линии в заданной метрике $\left\|g_{i j}\right\|$, имеют вид

$$
\frac{d^{2} x_{k}}{d s^{2}}+\sum_{i=1}^{n} \sum_{j=1}^{n} \Gamma_{i j}^{k} \frac{d x_{i}}{d s} \frac{d x_{j}}{d s}=0, \quad k=1,2, \ldots, n,
$$

где $s$-натуральный параметр (длина дуги). В нашем случае, пользуясь вычисленными символами Кристоффеля, мы можем записать эту систему в виде

$$
\begin{array}{ll}
\frac{d^{2} x_{k}}{d s^{2}}+\frac{K}{x_{n}} \frac{d x_{n}}{d s} \frac{d x_{k}}{d s}=0, & k=1, \ldots, n-1, \\
\frac{d^{2} x_{n}}{d s^{2}}-\frac{K}{2 x_{n}} \sum_{i=1}^{n}\left(\frac{d x_{i}}{d s}\right)^{2}+\frac{K}{2 x_{n}}\left(\frac{d x_{n}}{d s}\right)^{2}=0 . &
\end{array}
$$

Уравнения (22) могут быть записаны в виде

$$
x_{n}^{-K} \frac{d}{d s}\left(x_{n}^{K} \frac{d x_{k}}{d s}\right)=0, \quad k=1, \ldots, n-1 .
$$

Умножая (24) на $x_{n}^{K}$, интегрируя и деля на $x_{n}^{K}$, получим

$$
\frac{d x_{k}}{d s}=\frac{C_{k}}{x_{n}^{K}}, \quad k=1, \ldots, n-1 .
$$


Подставляя (25) в (23), имеем

$$
\frac{d^{2} x_{n}}{d s^{2}}-\frac{K B^{2}}{2 x_{n}^{2 K+1}}+\frac{K}{2 x_{n}}\left(\frac{d x_{n}}{d s}\right)^{2}=0,
$$

где $B$ определено выше. Уравнение (26) стандартным образом допускает понижение порядка. Положим $p=p\left(x_{n}\right)=d x_{n} / d s, v=p^{2}$; тогда $d^{2} x_{n} / d s^{2}=p^{\prime} p=v^{\prime} / 2$. После этого уравнение (26) сведется к виду

$$
v^{\prime}+\frac{K}{x_{n}} v=\frac{B^{2} K}{x_{n}^{2 K+1}} \quad \Longleftrightarrow \quad \frac{d}{d x_{n}}\left(x_{n}^{K} v\right)=\frac{B^{2} K}{x_{n}^{K+1}} .
$$

Интегрируя и деля на $x_{n}^{K}$, получим

$$
v=p^{2}=\left(\frac{d x_{n}}{d s}\right)^{2}=\frac{C_{n}}{x_{n}^{K}}-\frac{B^{2}}{x_{n}^{2 K}} .
$$

Известно (см. [10]), что геодезические линии обладают свойством

$$
\sum_{i=1}^{n} \sum_{j=1}^{n} g^{i j} \frac{d x_{i}}{d s} \frac{d x_{j}}{d s}=\text { const } .
$$

В рассматриваемом случае это приведет к равенству

$$
\sum_{i=1}^{n} x_{n}^{K}\left(\frac{d x_{i}}{d s}\right)^{2}=\text { const . }
$$

Из (21) легко выводится, что постоянная в равенстве $(27)$ совпадает с $C_{n}$.

\section{СПИСОК ЛИТЕРАТУРЫ}

1. Ибрагимов H. Х. Группы преобразований в математической физике. - М.: Наука, 1983.

2. Ильин B. А. О рядах Фурье по фундаментальным системам функций оператора Бельтрами// Диффер. уравн. - 1969. - 5, № 11. - С. 1940-1978.

3. Катрахов В. В., Ситник С. М. Метод преобразования и краевые задачи для сингулярных эллиптических уравнений// Совр. мат. Фундам. напр. - 2018. - 64, № 2. - С. 211-426.

4. Киприянов И. А. Преобразование Фурье-Бесселя и теоремы вложения для весовых классов// Тр. Мат. ин-та им. В. А. Стеклова РАН. - 1967. - 89. - С. 130-213.

5. Киприянов И. А. Сингулярные эллиптические краевые задачи. - М.: Наука, 1997.

6. Ляхов Л. Н. Весовые сферические функции и потнциалы Рисса, порожденные обобщенным сдвигом. - Воронеж: ВГТА, 1997.

7. Феллер В. О решениях линейных диференциальных уравнений в частных производных второго порядка эллиптического типа// Усп. мат. наук. - 1941. - № 8. - С. 232-248.

8. Хелгасон С. Дифференциальная геометрия и симметрические пространства. - М.: Мир, 1964.

9. Хелгасон С. Группы и геометрический анализ. - М.: Мир, 1987.

10. Эйзенхарт Л. Т. Риманова геометрия. - М.: ИЛ, 1948.

Половинкина Марина Васильевна

Воронежский государственный университет инженерных технологий

E-mail: polovinkina-marina@yandex.ru

Половинкин Игорь Петрович

Воронежский государственный университет

E-mail: polovinkin@yandex.ru 